\title{
Progress in the Development of a Continuous Adiabatic Demagnetization Refrigerator
}

\author{
Peter Shirron ${ }^{1}$, Edgar Canavan ${ }^{1}$, Michael DiPirro ${ }^{1}$, Michael Jackson ${ }^{1}$, \\ Todd King ${ }^{2}$ and James Tuttle ${ }^{1}$
}

\author{
${ }^{1}$ Code 552, ${ }^{2}$ Code 541 \\ NASA/Goddard Space Flight Center \\ Greenbelt, MD 20771
}

\begin{abstract}
We report on recent progress in the development of a continuous adiabatic demagnetization refrigerator (CADR). Continuous operation avoids the constraints of long hold times and short recycle times that lead to the generally large mass of single-shot ADRs, allowing us to achieve an order of magnitude larger cooling power per unit mass. Our current design goal is $10 \mu \mathrm{W}$ of cooling at $50 \mathrm{mK}$ using a $6-10 \mathrm{~K}$ heat sink. The estimated mass is less than $10 \mathrm{~kg}$, including magnetic shielding of each stage. The relatively high heat rejection capability allows it to operate with a mechanical cryocooler as part of a cryogen-free, low temperature cooling system. This has the advantages of long mission life and reduced complexity and cost. We have assembled a three-stage CADR and have demonstrated continuous cooling using a superfluid helium bath as the heat sink. The temperature stability is $8 \mu \mathrm{K}$ rms or better over the entire cycle, and the cooling power is $2.5 \mu \mathrm{W}$ at $60 \mathrm{mK}$ rising to $10 \mu \mathrm{W}$ at $100 \mathrm{mK}$.
\end{abstract}

\subsection{INTRODUCTION}

To meet the growing need for more capable low temperature refrigerators for space astronomy missions, we are developing an magnetic refrigerator that operates continuously at low temperature $[1,2]$. It is a multi-stage system that uses one stage to directly cool a load, and others to periodically cascade heat up to a heat sink. Since the heat transfer process does not disrupt cooling, it can be performed on a relatively short time scale ( $\sim$ hour $)$. This means each stage requires proportionally less refrigerant mass than a single-shot ADR [3] would need. We project that a CADR providing $10 \mu \mathrm{W}$ of cooling at $50 \mathrm{mK}$ using a $6-10 \mathrm{~K}$ heat sink will have a cold mass less than $10 \mathrm{~kg}$.

\subsection{DESIGN}

The design of an ADR depends most critically on the operating temperature, the cooling power and the heat sink temperature. For future space astronomy missions using large-format, low-temperature detectors, cooling powers of up to $10 \mu \mathrm{W}$ and temperatures as low as $50 \mathrm{mK}$ are expected to be needed. Heat sink temperatures will range from about $1 \mathrm{~K}$ to as high as $6-10 \mathrm{~K}$. This range reflects the possible pre-cooler options, from superfluid helium dewars to mechanical cryocoolers. The latter are now being baselined for a variety of upcoming missions, including Constellation-X, so it is important to begin developing low temperature coolers that can accommodate their use. The impact of such high heat rejection temperatures is actually quite 


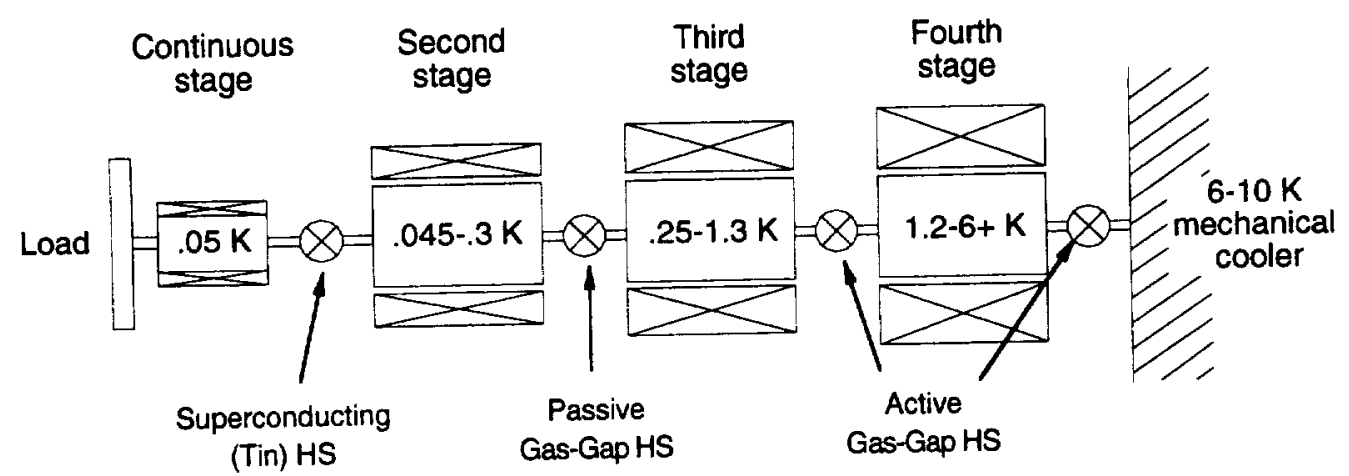

FIGURE 1. Schematic of a four-stage CADR capable of operating between $50 \mathrm{mK}$ and 6-10 K.

significant since it is virtually impossible for single-stage ADRs to operate over such a wide temperature range. The multi-stage architecture of our CADR, allows us to meet this requirement with relative ease. A linear chain of ADR stages, each typically spanning a factor of 5 in temperature, can in principle operate over an unlimited temperature range.

The preliminary design for a CADR capable of operating between $50 \mathrm{mK}$ and $6-10 \mathrm{~K}$ is shown in figure 1. Its four stages each consist of a cylindrical "salt pill" (containing the refrigerant), a magnet, magnetic shielding and a heat switch. The temperature range of each stage is determined by the properties of the heat switches. A superconducting tin switch is used between the continuous and second stages because it is one of the few types that can conduct well at temperatures below $100 \mathrm{mK}$. However its off conductance rises very rapidly with temperature, requiring the second stage to be kept below about $0.3 \mathrm{~K}$ at all times. Consequently the switch linking the second and third stages must be conductive at temperatures below $0.3 \mathrm{~K}$. This proved challenging, but ultimately possible for a passive ${ }^{3} \mathrm{He}$ gas-gap switch [4]. The third and fourth stages reject heat through conventional getter-activated ${ }^{3} \mathrm{He}$ gas-gap switches.

To date, we have constructed a three-stage CADR and tested it with a superfluid helium heat sink. The as-built refrigerant masses and magnetic field strengths shown in Figure 2. Stages 1 and 2 use chrome potassium alum (CPA) refrigerant because its entropy capacity is higher than ferric ammonium alum (FAA) at temperatures below $50 \mathrm{mK}$. Its low ordering temperature will

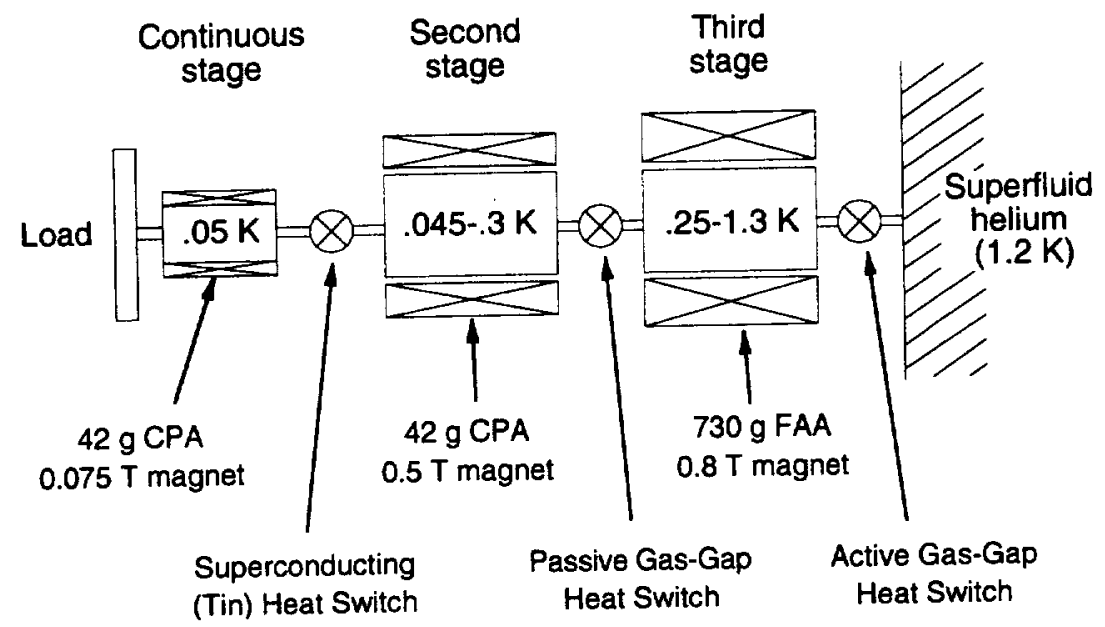

FIGURE 2. Schematic of a three-stage CADR with as-built component parameters. 


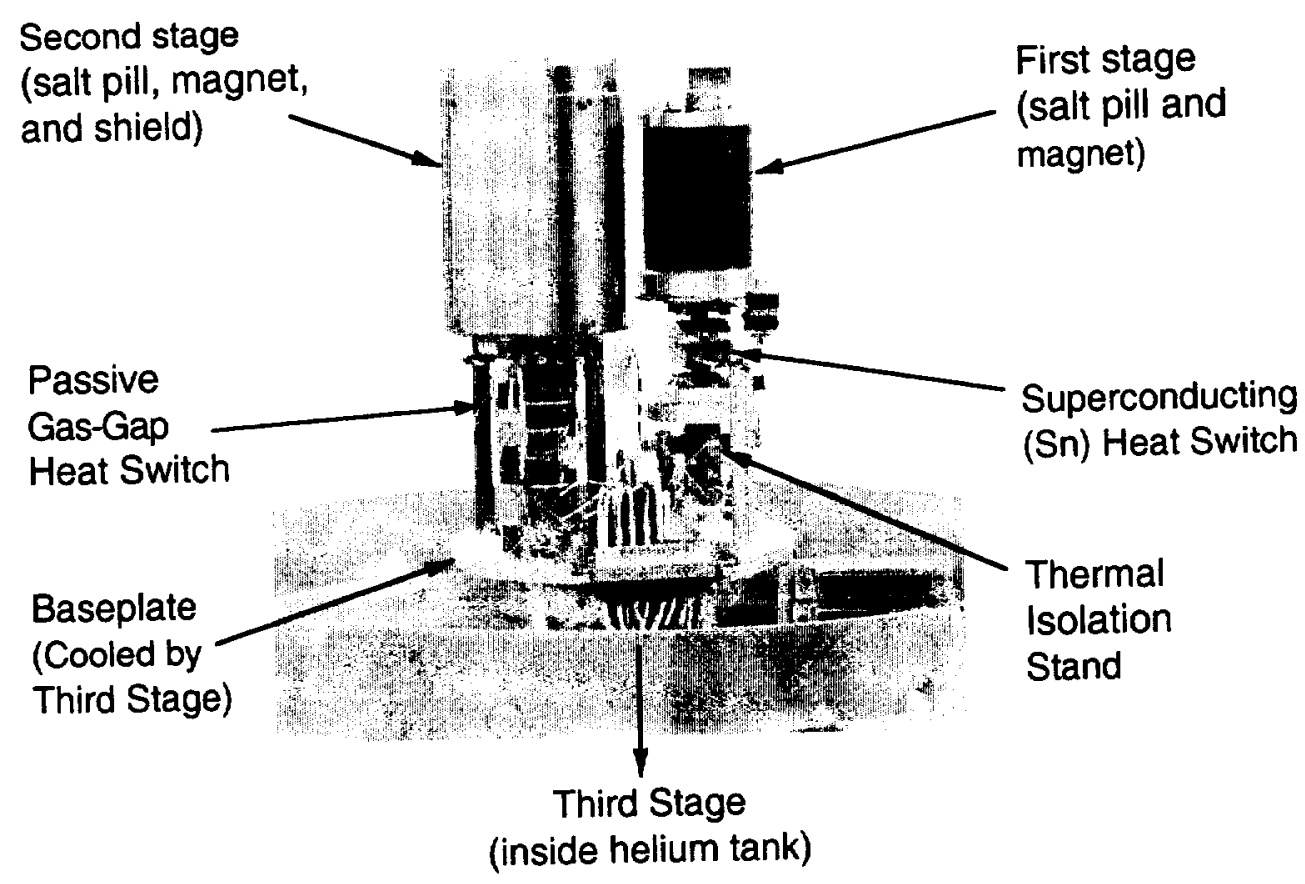

FIGURE 3. The first (right) and second (left) stages of a three-stage CADR in test configuration.

also allow the ADR to reach temperatures well below $50 \mathrm{mK}$ in the event that such capability is of practical use. The third stage, using ferric ammonium alum (FAA), is an engineering unit ADR produced for the X-Ray Spectrometer mission [3], and is much larger than necessary. In the next version of the CADR, it will be replaced by an $80 \mathrm{~g}$ CPA salt pill, identical to the second stage salt pill, and a $1.5 \mathrm{~T}$ magnet. Figure 3 is a picture of the CADR in the test dewar. The third stage and its magnet are located inside the helium tank of the dewar and are therefore out of sight beyond the bottom of the picture.

\subsection{OPERATION}

The CADR has two operational modes. The first involves cooling down from the heat sink temperature and establishing temperature control. The second is the periodic cycling of the upper stages to maintain constant temperature.

To begin, all stages are magnetized to full field with the heat switches turned on. Starting with the third, each stage is sequentially demagnetized (after turning the appropriate heat switch off) to the low end of its operating range. The rate is not critical but should be slow enough to efficiently cool the lower stages. In the final state, the continuous stage is near full capacity and is actively cooling the load. A control routine then takes over to automatically recycle each stage as needed.

Recycling consists of demagnetizing the adjacent upper stage to a lower temperature and closing the heat switch. The temperature controller automatically magnetizes the lower stage as the upper stage absorbs its heat. When the magnetic field reaches an upper threshold, the heat switch is opened, and the upper is magnetized to the high end of its range to reject the heat to the next stage. The process is repeated until the uppermost stage rejects heat to the heat sink. 


\subsection{CADR PERFORMANCE}

The key performance areas for a low temperature cooler are operating temperature, temperature stability, cooling power and efficiency.

\subsection{Operating Temperature}

To date, the CADR has operated continuously at temperatures down to $35 \mathrm{mK}$ and up to 200 $\mathrm{mK}$. Higher temperature operation is possible, but because of minor constraints imposed by the passive gas-gap heat switch, some software changes would be necessary in order to preserve high efficiency. These will be implemented as the need arises.

\subsection{Temperature Stability}

Temperature control is more of a concern for the CADR because of the need to periodically reverse heat flows into and out of the first stage. The control software minimizes the disturbances by such techniques as only turning the superconducting heat switch on and off when the temperature difference between the first and second stage is close to zero, and limiting the rate at which the heat flows can change. Another important consideration is for the temperature controllers to have fairly high bandwidth $(\sim 10 \mathrm{~Hz})$. With both analog and digital controllers the CADR can achieve control that is limited only by the noise of the temperature readout. Figure 4 shows the temperature and magnetic field of the continuous stage during one cycle conducted at $100 \mathrm{mK}$. The magnetic field has the characteristic sawtooth pattern as heat is alternately absorbed and rejected, however there is no apparent correlation between temperature fluctuations and any recycling events. The noise is $8 \mu \mathrm{K}$ rms or better throughout the cycle.

\subsection{Cooling Power}

The CADR's cooling power shown in Table 1. The cooling power is the maximum sustainable heat load that can be applied to the continuous stage. Although the CADR can tolerate larger momentary loads, when the heat load remains in excess of these values, the

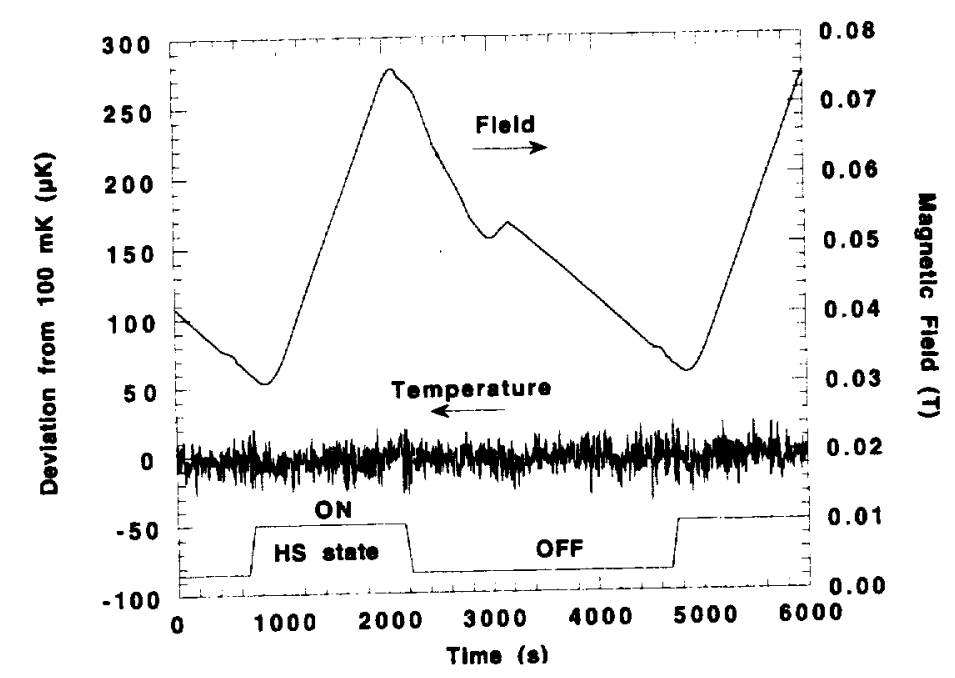

FIGURE 4. Temperature and magnetic field of the continuous stage during a complete cycle. 
TABLE 1. Demonstrated Cooling Power.

\begin{tabular}{cc} 
Temperature $(\mathbf{K})$ & Maximum Sustained Heat Load $(\mu \mathbf{W})$ \\
\hline 0.10 & 15 \\
0.09 & 13 \\
0.08 & 11 \\
0.06 & 7 \\
0.05 & 5 \\
0.035 & 1.5 \\
\hline
\end{tabular}

continuous stage eventually runs out of cooling capacity and loses temperature control. The heat load is approximately linear in temperature above $50 \mathrm{mK}$. The non-linearity below $50 \mathrm{mK}$ is due to several factors, the most important of which appear to be the rapidly increasing thermal boundary resistance between the salt and the thermal bus (which significantly reduces heat transfer efficiency), and reduced entropy capacity as the ordering temperature is approached.

\subsection{Thermodynamic Efficiency}

We have made careful measurements of the CADR's efficiency at both 50 and $100 \mathrm{mK}$ with applied heat loads as given in Table 1. The measurement includes all parasitic effects (such as eddy current heating, magnet and magnetic shield hysteresis, heat switch and suspension system parasitics, and thermal gradients within the salt pills) except for the following: we have neglected heat that is dissipated in the third stage magnet as well as the heat applied to turn on the third stage heat switch, and we have neglected dissipation in the room temperature electronics. The reason is that these components are not yet representative of the final design, and the dissipation is consequently much higher than we eventually expect. With this in mind, we regard the measurements of $50 \%$ efficiency at $100 \mathrm{mK}$ and $12 \%$ at $50 \mathrm{mK}$ as being the right order of magnitude for a complete system. Granted the efficiency will decrease when the neglected terms are added, but there is also some room for improvement, for example by thermally anchoring all magnets and shields to the heat sink rather than to the third stage, as is presently the case for the second stage magnet.

\subsection{SUMMARY}

We have constructed a three-stage CADR that operates continuously at temperatures down to $60 \mathrm{mK}$ with high cooling power. The system achieves $8 \mu \mathrm{K} \mathrm{rms}$ temperature stability throughout its cycling operations, and cooling powers of $2.5 \mu \mathrm{W}$ at $60 \mathrm{mK}$ rising to $10 \mu \mathrm{W}$ at $100 \mathrm{mK}$. In the near term we will construct a fourth stage that will allow the system to operate with a $4.2 \mathrm{~K}$ helium bath or eventually with a mechanical cooler running at 6-10 K. We are also working on designs for suspension systems and magnetic shielding so that the system can be packaged more compactly. Our present layout for a four-stage cooler operating with a $4.2 \mathrm{~K}$ heat sink will fit within a 2 -liter volume and weigh less than $7 \mathrm{~kg}$.

\subsection{ACKNOWLEDGMENTS}

This work was supported by NASA's Cross-Enterprise Technology Development Program and GSFC's Commercial Technology Development Program. 


\subsection{REFERENCES}

1. Shirron, P.J., Canavan, E.R., DiPirro, M.J., Tuttle, J.G., and Yeager, C.J., "A Multi-Stage Continuous Duty Adiabatic Demagnetization Refrigerator," in Adv. Cryo. Eng. 45B, edited by Q.-S. Shu et al., Plenum, New York, 2000, pp. 1629-1638.

2. Shirron, P.J., Canavan, E.R., DiPirro, M.J., Jackson, M., King, T., Panek, J., and Tuttle, J.G., "A Compact, High-Performance Continuous Magnetic Refrigerator for Space Missions", presented at the 2001 Space Cryogenics Workshop in Milwaukee, WI, accepted for publication in Cryogenics.

3. For descriptions of one- and two-stage ADRs, see Serlemitsos, A.T., SanSebastian, M., and Kunes, E., Cryogenics 32, 117 (1992), and Hagmann, C. and Richards, P.L., Cryogenics 34, 221 (1994).

4. Shirron, P.J., Canavan, E.R., DiPirro, M.J., Jackson, M., Panek, J., and Tuttle, J.G., "Passive Gas-Gap Heat Switches for Use in Adiabatic Demagnetization Refrigerators," presented at the 2001 Cryogenic Engineering Conference, Madison, WI, accepted for publication in Adv. Cryo. Eng. 47. 


\section{GSFC STI PUBLIC DISCLOSURE EXPORT CONTROL CHECKLIST}

The Export Control Office requests your assistance in assuring that your proposed disclosure of NASA scientific and technical information (STI) complies with the Export Administration Regulations (EAR, 15 CFR 730-774) and the International Traffic in Arms Regulations (ITAR, 22 CFR 120-130). The NASA Export Control Program requires that every domestic and international presentation/publication of GSFC STI be reviewed through the GSFC Export Control Office in accordance with the NASA Form 1676, NASA Scientific and Technical Documents Availability Authorization (DAA) process. Release of NASA information into a public forum may provide countries with interests adverse to the United States with access to NASA technology. Failure to comply with the ITAR regulations and/or the Commerce Department regulations may subject you to fines of up to $\$ 1$ million and/or up to 10 years imprisonment per violation. Completion of this checklist should minimize delays in approving most requests for presentation/publication of NASA STI.

Generally, the export of information pertaining to the design, development, production, manufacture, assembly, operation, repair, testing, maintenance or modification of defense articles, i.e., space flight hardware, ground tracking systems, launch vehicles to include sounding rockets and meteorological rockets, radiation hardened hardware and associated hardware and engineering units for these items are controlled by the State Department under the ITAR. A complete listing of items covered by the ITAR can be accessed at hup gstc-bluenun. gstc.nasa.gov export/regsitar.htm. The export of information with respect to ground based sensors, detectors, high-speed computers, and national security and missile technology items are controlled by the U.S. Commerce Department under the EAR. If the information intended for release falls within the above categories but otherwise fits into one or more of the following exemptions, the information may be released.

\section{EXEMPTION I}

If your information is already in the public domain in its entirety through a non-NASA medium and/or through NASA release previously approved by the Export Control Office, the information is exempt from further review. If the information falls into this category, you may attest that you are using this exemption by signing below.

Signature

$\overline{\text { Date }}$

\section{EXEMPTION II}

If your information pertains exclusively to the release of scientific data, i.e., data pertaining to studies of clouds, soil, vegetation, oceans, and planets, without the disclosure of information pertaining to articles controlled by the ITAR or EAR, such as flight instruments, high speed computers, or launch vehicles, the information is exempt from further review. If the information falls into this category, you may attest that you are using this exemption by signing below. 


\section{EXEMPTION III}

If your information falls into the areas of concern as referenced above, but is offered at a general purpose or high level, i.e. poster briefs and overviews, where no specific information pertaining to ITAR or EAR controlled items is offered, the information is exempt from further review. If the information falls into this çategory, you may attest that you are using this exemption by signing below.
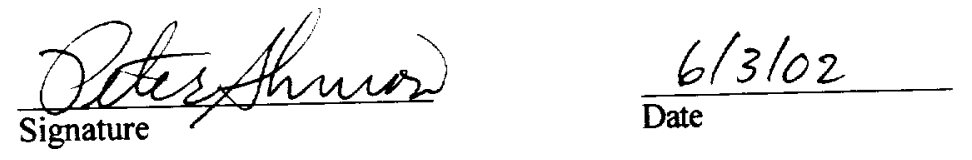

\section{EXEMPTION IV}

If your information is not satisfied by the three exemptions stated above, the information may be released using exemption 125.4(b)(13) of the ITAR. Use of this exemption is afforded only to agencies of the Federal Government and allows the release of ITAR controlled information into the public domain. The GSFC Export Control Office has determined that use of this exemption will be allowed only after receiving assurance that such release is a responsible action. The following internal guideline has been established regarding use of this authority: That the information does not offer specific insight into the design, development, production, or manufacture of an identified ITAR controlled item (reference paragraph 2 , above) in sufficient detail (by itself or in conjunction with other publications) to allow potential adversaries to replicate the item or exploit or defeat controlled U.S. technologies. All signatures of approval on NASA Form 1676 expressly indicate concurrence with the responsible use of Exemption IV when Exemption IV has been cited by the author. If you determine that you have met this criteria, you may attest your determination by signing below, and the GSFC Export Control Office will offer favorable consideration toward approving your presentation/publication request under this special exemption.

Signature

Date

If you do not satisfy the above exemptions, please contact the GSFC Export Control Office for further clarification on the releasability of your information under the ITAR or EAR. 